\title{
Reply to the comment on: "Does an atom interferometer test the gravitational redshift at the Compton frequency?"
}

\author{
Peter Wolf, ${ }^{1}$ Luc Blanchet, ${ }^{2}$ Christian J. Bordé, ${ }^{1,3}$ Serge \\ Reynaud, ${ }^{4}$ Christophe Salomon, ${ }^{5}$ and Claude Cohen-Tannoudji ${ }^{5}$ \\ ${ }^{1}$ LNE-SYRTE, CNRS UMR 8630, UPMC, Observatoire de Paris, \\ 61 avenue de l'Observatoire, 75014 Paris, France \\ ${ }^{2}$ GRECO, Institut d'Astrophysique de Paris, CNRS UMR 7095, \\ UPMC, $98^{\text {bis }}$ boulevard Arago, 75014 Paris, France \\ ${ }^{3}$ Laboratoire de Physique des Lasers, CNRS UMR 7538, \\ 99 avenue Jean-Baptiste Clément, 93430 Villetaneuse, France \\ ${ }^{4}$ Laboratoire Kastler Brossel, CNRS UMR 8552, ENS, UPMC, Campus Jussieu, 75252 Paris, France \\ ${ }^{5}$ Laboratoire Kastler Brossel et Collège de France, CNRS UMR 8552, \\ ENS, UPMC, 24 rue Lhomond, 75231 Paris, France
}

(Dated: August 29, 2018)

\begin{abstract}
Hohensee, Chu, Peters and Müller have submitted a comment (arXiv:1112.6039 [gr-qc]) on our paper "Does an atom interferometer test the gravitational redshift at the Compton frequency?", Classical and Quantum Gravity 28, 145017 (2011), arXiv:1009.2485 [gr-qc]. Here we reply to this comment and show that the main result of our paper, namely that atom interferometric gravimeters do not test the gravitational redshift at the Compton frequency, remains valid.
\end{abstract}

In their comment Hohensee, Chu, Peters and Müller (referred to as HCPM here) 1] re-affirm their earlier interpretation, based on an analogy with classical clock experiments, of atom interferometric gravimeters as testing the gravitational redshift. They repeat previous arguments, in particular the cancellation between the time dilation and laser phase contributions to the phase shift, and arguments centered around Schiff's conjecture. Here we re-affirm that these arguments are incorrect or irrelevant, as shown below, and that the main result of our paper [2], namely that atom interferometric gravimeters do not test the gravitational redshift at Compton's frequency, remains valid.

First HCPM argue that the absence of the Compton frequency (or equivalently the atom's mass) in the final phase shift, does not prevent the experiment from being a redshift test at that frequency. They claim that this is also the case for redshift tests using standard clocks, like the Pound-Rebka experiment, where the redshift effect is cancelled by a controlled first order Doppler effect, thus effectively leaving a null signal independent of the $14.4 \mathrm{keV}$ transition frequency. This analogy is fallacious : the Pound-Rebka experiment is based on a resonance between atoms sitting at the top and bottom of a tower. The controlled Doppler shift is just a clever experimental method for scanning the resonance which is affected by the redshift (cf. Figure 1. in [3]). The resulting signal is thus null on the exact center of the resonance curve but not at other frequencies. In the atom gravimeter in contrast, the cancellation of the Compton frequency is inherent to the interferometer : the action phase shift involving the Compton frequency is always zero as shown in [2] and the measurement of that frequency (and of the associated redshift) is intrinsically impossible. Furthermore it is manifestly incorrect to state as HCPM do that the cancellation of the clock frequency is a generic feature of any redshift measurement. Most clock experiments [4, [5] measure frequencies of two electromagnetic signals delivered by two clocks. The redshift effect is thus associated to a relation such as $\Delta f=f_{0} \Delta U / c^{2}$ where $\Delta f$ is the measured frequency difference and $f_{0}$ the common proper frequency of the clocks. This could also be done in principle for a Pound-Rebka-like experiment if experimental means (say frequency combs) were available for accurate measurements of frequencies in the X-ray domain. By contrast, in the atomic interferometer considered by HCPM, there is no real emission of a photon at the Compton frequency and no measurement of the frequency of this photon by a detector.

The interferometer considered by HCPM is in fact not an atomic clock. The two states $g$ and $g^{\prime}$ appear symmetrically in the two arms of the interferometer and no resonance is obtained when one varies the laser frequency. As explained in [2], there are other non-symmetric atomic interferometers where the output signal exhibits resonances when the laser frequency is varied. They provide a measurement of the atomic frequency $\omega_{g g^{\prime}}$ (see discussion after (2.21) in [2]), so that these interferometers can be also considered as atomic clocks oscillating at $\omega_{g g^{\prime}}$. To get an oscillation at the Compton frequency, one would need to have in the two arms of the interferometer two states, whose energies differ by $m c^{2}$, which is far beyond present day technology. Furthermore, to test the red shift at Compton frequency, two different clocks of this type located at two different altitudes would have to be built and their frequencies compared.

In a related argument (eq. (1) and text below in their comment), HCPM restate their previous claim that in the total phase shift the time dilation phase $\phi_{t}$ cancels the laser phase $\phi_{\text {laser }}$ and not the gravitational phase $\phi_{r}$, whereas we have demonstrated in [2] that the free evolution phase $\phi_{S}=\phi_{r}+\phi_{t}$ is necessarily zero for all closed paths that 
follow trajectories obtained from the same Lagrangian as the one used for the phase integral. The latter fact implies that the difference of action integrals is zero and that the Compton frequency and redshift at that frequency are unmeasurable, no matter what modified Lagrangian is used (i.e. independently of any redshift violating parameter $\beta)$. We re-affirm that the fundamental cancellation is between $\phi_{r}$ and $\phi_{t}$, one reason being that $\phi_{S}=\phi_{r}+\phi_{t}=0$ is an invariant statement, since $\phi_{S}$ is the contour integral of the proper time (see first term of the right-hand side of (1.3) in [2]) which is invariant under coordinate transformations. By contrast the statement that $\phi_{t}+\phi_{\text {laser }}=0$ is not invariant and is only true in the rest frame of the lasers. To see this in a simple example, analyze the atom gravimeter in a frame that is freely falling in the Earth's gravitational field. An elementary calculation shows that $\phi_{r}$ and $\phi_{t}$ are separately zero in that frame, $\phi_{r}=\phi_{t}=0$, but that $\phi_{\text {laser }}=k g T^{2}$, where $k$ is the laser wavevector and $T$ the interrogation time, because the lasers are accelerated upwards in that frame. Thus there is no cancellation between $\phi_{t}$ and $\phi_{\text {laser }}$ in that frame but still we have $\phi_{S}=\phi_{r}+\phi_{t}=0$.

Concerning Schiff's conjecture, we do not agree with the analysis of HCPM. Schiff's conjecture states that any violation of the universality of free fall (UFF) also implies a violation of the universality of clock rates (UCR) and vice versa. Does this mean that it makes no sense to distinguish between the two types of tests ? Our answer is "No", because the relation between UFF and UCR tests depends on the experiments under consideration, on the nuclear or atomic models used for the analysis of the clocks and the test masses, and on the particular model used for the violation of UFF and UCR.

The next question is then whether the atom gravimeter measurement is more directly comparable to UFF or UCR tests? As shown in [2] but also e.g. in [6] the answer is clearly UFF as it constrains the same parameters as other UFF tests, whereas it requires nuclear and atomic models to relate it to parameters measured in UCR tests. To make this point more explicit, let us quote the SME analysis presented in [7] : the UFF test of [8] sets a limit on $\beta^{\mathrm{Be}}-\beta^{\mathrm{Al}}$ (to leading order), the atom gravimeter test sets a limit on $\beta^{\text {Cs }}-\beta^{\text {grav }}$ (where "grav" stands for the test mass in the classical gravimeter) and the Pound-Rebka experiment on $\xi_{57}^{\mathrm{Moss}}-\beta^{\text {grav }}$. To relate the former two one only requires the atomic composition of Be, $\mathrm{Al}$ and grav (equ. (8) of [7]) but to relate them to the Pound-Rebka measurement one additionally requires a nuclear model for the $14.4 \mathrm{keV}$ transition in ${ }^{57} \mathrm{Fe}$ (see text before (11) in [7]).

To conclude, let us briefly recall the compelling argument (see e.g. [6]) that atom interferometers test the UFF. This argument is agreed on since the realization of the first atomic gravimeters two decades ago [9]. The atoms with inertial mass $m_{i}$ and gravitational mass $m_{g}$ obey the Lagrangian $L=\frac{1}{2} m_{i} \dot{z}^{2}-m_{g} g z$ in the gravitational field of the Earth. The phase shift is the sum of the free evolution phase given by the difference of action integrals $\phi_{S}$ along the two paths, and of the contribution $\phi_{\text {laser }}$ coming from the interaction with the lasers. The free evolution phase $\phi_{S}$ is exactly zero for a closed total path (see e.g. [2]), and the phase shift reduces to $\phi_{\text {laser }}=\left(m_{g} / m_{i}\right) g k T^{2}$ using the position of the atoms deduced from the Lagrangian. Comparing with the measurement by a nearby classical gravimeter of the gravitational acceleration of a freely falling macroscopic mass (corner cube), $g_{\text {meas }}=\left(M_{g} / M_{i}\right) g$, one obtains to first order $\phi_{\text {laser }}=(1+\eta) g_{\text {meas }} k T^{2}$ where $\eta=m_{g} / m_{i}-M_{g} / M_{i}$ is the Eötvos parameter between the atoms and the corner cube, showing that measurement of the phase shift gives a test on possible violations of the UFF between atoms and macroscopic masses.

[1] M.A. Hohensee, S. Chu, A. Peters and H. Müller, Comment on: "Does an atom interferometer test the gravitational redshift at the Compton frequency?", arXiv:1112.6039 [gr-qc].

[2] P. Wolf, L. Blanchet, Ch.J. Bordé, S. Reynaud, C. Salomon, and C. Cohen-Tannoudji, Class. Quant. Grav. 28, 145017 (2011), arXiv:1009.2485 [gr-qc].

[3] R.V. Pound and J.L. Snider, Phys. Rev. 140, B788 (1965).

[4] R. Vessot and M. Levine, Gen. Rel. and Grav. 10, 181 (1979).

[5] L. Cacciapuoti and C. Salomon, Eur. Phys. J. Spec. Top. 127, 57 (2009).

[6] D. Giulini (2011), arXiv:1105.0749 [gr-qc].

[7] M.A. Hohensee, S. Chu, A. Peters and H. Müller, Phys. Rev. Lett. 106, 151102 (2011).

[8] S. Schlamminger, K.-Y. Choi, T. Wagner, J. Gundlach, and E. Adelberger, Phys. Rev. Lett. 100, 041101 (2008).

[9] M. Kasevich and S. Chu, Phys. Rev. Lett. 67, 181 (1991). 